Supporting Information Available: Copies of the ${ }^{1} \mathrm{H}-\mathrm{NMR},{ }^{13} \mathrm{C}$ NMR and/or Mass spectra and analytical data of compounds $2,3,4-B n$, and 4-Me.

\title{
Methyl 2,3-di-O-benzyl-D-ribofuranoside (2)
}

2,3-Di- $O$-benzyl-D-ribofuranoside (2) was prepared according to the general method from D-ribose $(1.50 \mathrm{~g}, 10.0 \mathrm{mmol})$ in $88 \%$ (3.03 g) overall yield in four steps via methyl Dribofuranoside, methyl 5-O-4,4'-dimethoxytrityl(DMTr)-D-ribofuranoside, and 2,3-di-Obenzyl-5-O-DMTr-D-ribofuranoside. $\alpha$-anomer: ${ }^{1} \mathrm{H}-\mathrm{NMR}\left(\mathrm{CDCl}_{3}\right): \delta$ 7.2-7.4 $(10 \mathrm{H}, \mathrm{m}, \mathrm{Ph}-$ $\mathrm{H}), 4.88\left(1 \mathrm{H}, \mathrm{d}, \mathrm{J}_{1,2}=4.2 \mathrm{~Hz}, \mathrm{H1}\right), 4.76(1 \mathrm{H}, \mathrm{d}, \mathrm{J}=12.6 \mathrm{~Hz}, \mathrm{Bn}-\mathrm{CH}), 4.66(2 \mathrm{H}, \mathrm{s}, \mathrm{Bn}-\mathrm{CH} \mathrm{x}$ 2), $4.59(1 \mathrm{H}, \mathrm{d}, \mathrm{J}=12.6 \mathrm{~Hz}, \mathrm{Bn}-\mathrm{CH}), 4.18(1 \mathrm{H}, \mathrm{m}, \mathrm{H} 4), 3.85\left(1 \mathrm{H}, \mathrm{dd}, \mathrm{J}_{2,3}=6.9 \mathrm{~Hz}, \mathrm{~J}_{3,4}=\right.$ $3.7 \mathrm{~Hz}, \mathrm{H} 3), 3.74\left(1 \mathrm{H}, \mathrm{dd}, \mathrm{J}_{1,2}=4.2 \mathrm{~Hz}, \mathrm{~J}_{2,3}=6.9 \mathrm{~Hz}, \mathrm{H} 2\right), 3.67(1 \mathrm{H}, \mathrm{m}, \mathrm{H} 5$ ') $3.47(3 \mathrm{H}, \mathrm{s}$, $\left.\mathrm{OCH}_{3}\right), 3.43(1 \mathrm{H}, \mathrm{m}, \mathrm{H} 5), 1.57(1 \mathrm{H}, \mathrm{dd}, \mathrm{J}=4.7 \mathrm{~Hz}, \mathrm{~J}=8.1 \mathrm{~Hz}, 5-\mathrm{OH}){ }^{13} \mathrm{C}-\mathrm{NMR}\left(\mathrm{CDCl}_{3}\right)$ : $138.21,137.77,128.31,128.08,127.91,127.77,127.69,102.63,82.98,78.15,74.80$, 72.62, 72.54, 62.63, 55.44. Anal. calcd for $\mathrm{C}_{20} \mathrm{H}_{24} \mathrm{O}_{5}$ : C, 69.75; H, 7.02. Found: C, 69.45; H, 6.97. $\beta$-anomer: ${ }^{1} \mathrm{H}-\mathrm{NMR}\left(\mathrm{CDCl}_{3}\right): \delta$ 7.2-7.4 $(10 \mathrm{H}, \mathrm{m}, \mathrm{Ph}-\mathrm{H}), 4.89(1 \mathrm{H}, \mathrm{s}, \mathrm{H} 1), 4.66$ $(1 \mathrm{H}, \mathrm{d}, \mathrm{J}=12.1 \mathrm{~Hz}, \mathrm{Bn}-\mathrm{CH}), 4.63(1 \mathrm{H}, \mathrm{d}, \mathrm{J}=12.1 \mathrm{~Hz}, \mathrm{Bn}-\mathrm{CH}), 4.59(1 \mathrm{H}, \mathrm{d}, \mathrm{J}=11.7 \mathrm{~Hz}$ Bn-CH), $4.50(1 \mathrm{H}, \mathrm{d}, \mathrm{J}=11.7 \mathrm{~Hz}, \mathrm{Bn}-\mathrm{CH}), 4.28(1 \mathrm{H}, \mathrm{m}, \mathrm{H} 4), 4.13\left(1 \mathrm{H}, \mathrm{dd}, \mathrm{J}_{2,3}=4.9 \mathrm{~Hz}\right.$, $\left.\mathrm{J}_{3,4}=7.0 \mathrm{~Hz}, \mathrm{H} 3\right), 3.74\left(1 \mathrm{H}, \mathrm{d}, \mathrm{J}_{2,3}=4.9 \mathrm{~Hz}, \mathrm{H} 2\right), 3.80$ (1H, m, H5'), 3.58 (1H, m, H5), 3.37 $\left(3 \mathrm{H}, \mathrm{s}, \mathrm{OCH}_{3}\right), 1.88(1 \mathrm{H}, \mathrm{dd}, \mathrm{J}=4.1 \mathrm{~Hz}, \mathrm{~J}=8.6 \mathrm{~Hz}, 5-\mathrm{OH}){ }^{13} \mathrm{C}-\mathrm{NMR}\left(\mathrm{CDCl}_{3}\right): \delta 137.76$, $128.43,127.96,127.86,106.91,82.33,80.28,77.35,72.69,72.51,62.73,55.56$. Anal. calcd for $\mathrm{C}_{20} \mathrm{H}_{24} \mathrm{O}_{5}:$ C, 69.75; H, 7.02. Found: C, 69.45; H, 6.97.

\section{4,5-Di-O-acetyl-2,3-di- $O$-benzyl-D-ribose diethyl dithio acetal (3).}

Compound $2(5.28 \mathrm{~g}, 15.4 \mathrm{mmol})$ was dissolved in $\mathrm{c} . \mathrm{HCl}(15 \mathrm{~mL})$ and ethanethiol $(15 \mathrm{~mL})$ 
at $0{ }^{\circ} \mathrm{C}$ and stirred for $3 \mathrm{~h}$. To the reaction solution was added $\mathrm{Et}_{2} \mathrm{O}(20 \mathrm{~mL})$, and then neutralized with sodium hydrogencarbonate. The resulting salt was filtrated and filtrate was evaporated. The residue was partitioned between $\mathrm{H}_{2} \mathrm{O}(100 \mathrm{~mL})$ and $\mathrm{Et}_{2} \mathrm{O}(200 \mathrm{~mL}$ x 1 , $100 \mathrm{~mL} \times 2)$. The combined $\mathrm{Et}_{2} \mathrm{O}$ layer was washed with brine $(100 \mathrm{~mL})$ and dried $\left(\mathrm{MgSO}_{4}\right)$. The organic layer was filtered and concentrated to dryness. To the resulting residue in dichloromethane $\left(\mathrm{CH}_{2} \mathrm{Cl}_{2}\right)(150 \mathrm{~mL})$ under an argon atmosphere DMAP $(7.89 \mathrm{~g}, 64.7$ mmol) and acetic anhydride $(3.6 \mathrm{~mL}, 38.5 \mathrm{mmol})$ were added, then a solution was stirred for overnight. The reaction mixture was quenched with $\mathrm{MeOH}$ and then was partitioned between $\mathrm{Et}_{2} \mathrm{O}(200 \mathrm{~mL} \times 1,100 \mathrm{~mL} \times 2)$ and $5 \%$ citric acid aqueous $(100 \mathrm{~mL})$. $\mathrm{An}^{\mathrm{E}} \mathrm{t}_{2} \mathrm{O}$ layer was washed with a saturated aqueous $\mathrm{NaHCO}_{3}$ and brine. The organic layer was dried $\left(\mathrm{MgSO}_{4}\right)$, and concentrated to dryness. The residue was subjected to chromatography on a column of silica gel employing hexane- AcOEt system to give 3 (7.48 g, $93 \%)$. ${ }^{1} \mathrm{H}-\mathrm{NMR}$ $\left(\mathrm{CDCl}_{3}\right): \delta$ 7.2-7.5 $(10 \mathrm{H}, \mathrm{m}, \mathrm{Ph}-\mathrm{H}), 5.58\left(1 \mathrm{H}, \mathrm{ddd}, \mathrm{J}_{3,4}=2.3 \mathrm{~Hz}, \mathrm{~J}_{4,5}=7.9 \mathrm{~Hz}, \mathrm{~J}_{4,5}=3.0 \mathrm{~Hz}\right.$ H4), $4.97(1 \mathrm{H}, \mathrm{d}, \mathrm{J}=11.0 \mathrm{~Hz}, \mathrm{Bn}-\mathrm{CH}), 4.77(1 \mathrm{Hd}, \mathrm{J}=11.1 \mathrm{~Hz}, \mathrm{Bn}-\mathrm{CH}), 4.76(1 \mathrm{H}, \mathrm{d}, \mathrm{J}=$ $10.9 \mathrm{~Hz}, \mathrm{Bn}-\mathrm{CH}), 4.58(1 \mathrm{H}, \mathrm{d}, \mathrm{J}=11.1 \mathrm{~Hz}, \mathrm{Bn}-\mathrm{CH}), 4.37\left(1 \mathrm{H}, \mathrm{dd}, \mathrm{J}_{4,5^{\circ}}=3.0 \mathrm{~Hz}, \mathrm{~J}_{5,5^{\circ}}=12.3\right.$ Hz, H5'), $4.21\left(1 \mathrm{H}, \mathrm{dd}, \mathrm{J}_{4,5}=7.9 \mathrm{~Hz}, \mathrm{~J}_{5,5^{\circ}}=12.3 \mathrm{~Hz}, \mathrm{H} 5\right), 4.16\left(1 \mathrm{H}, \mathrm{d}, \mathrm{J}_{1,2}=3.2 \mathrm{~Hz}, \mathrm{H1}\right)$, $4.06\left(1 \mathrm{H}, \mathrm{dd}, \mathrm{J}_{2,3}=7.6 \mathrm{~Hz}, \mathrm{~J}_{3,4}=2.3 \mathrm{~Hz}, \mathrm{H} 3\right), 3.91\left(1 \mathrm{H}, \mathrm{dd}, \mathrm{J}_{1,2}=3.2 \mathrm{~Hz}, \mathrm{~J}_{2,3}=7.6 \mathrm{~Hz}, \mathrm{H} 2\right)$, 2.5-2.8 (4H, m, $\left.\mathrm{SC}_{2} \mathrm{CH}_{3} \times 2\right), 2.08\left(3 \mathrm{H}, \mathrm{s}, \mathrm{COCH}_{3}\right), 2.00\left(3 \mathrm{H}, \mathrm{s}, \mathrm{COCH}_{3}\right), 1.24(3 \mathrm{H}, \mathrm{t}, \mathrm{J}=$ $\left.7.4 \mathrm{~Hz}, \mathrm{SCH}_{2} \underline{\mathrm{C}}_{3}\right), 1.20\left(3 \mathrm{H}, \mathrm{t}, \mathrm{J}=7.4 \mathrm{~Hz}, \mathrm{SCH}_{2} \underline{\mathrm{C}}_{3}\right) .{ }^{13} \mathrm{C}-\mathrm{NMR}\left(\mathrm{CDCl}_{3}\right): \delta 170.63$, $169.79,137.63,137.57,128.34,128.22,128.15,128.02,127.80,127.64,81.93,79.60$, 74.71, 73.42, 71.83, 63.32, 53.44, 26.30, 24.96, 21.02, 20.74, 14.35, 14.31 Anal. calcd for $\mathrm{C}_{27} \mathrm{H}_{36} \mathrm{O}_{6} \mathrm{~S}$ : C, 62.28; H, 6.97. Found: C, 62.39; H, 6.99. 


\section{2,3-Di-O-benzyl-D-ribose dibenzyl acetal (4-Bn).}

Compound 4-Bn $(2.87 \mathrm{~g}, 4.7 \mathrm{mmol})$ was prepared by the general deacetyration of 4,5-di- $O$ acetyl-2,3-di- $O$-benzyl-D-ribose dibenzyl acetal that was synthesized according to the report of Serfontein et al. ${ }^{8}$

4,5-Di- $O$-acetyl-2,3-di- $O$-benzyl-D-ribose dibenzyl acetal .

Yellow mercuric oxide $(648 \mathrm{mg}, 3.0 \mathrm{mmol})$ and calcium sulfate- $2 \mathrm{H}_{2} \mathrm{O}(3.6 \mathrm{~mL}, 38.5$ mmol) were added to a solution of $\mathbf{3}(520 \mathrm{mg}, 1.0 \mathrm{mmol})$ in anhydrous benzyl alcohol (5 $\mathrm{mL})$ under an argon atmosphere at $\mathrm{rt}$. A mercuric chloride (680 $\mathrm{mg}, 2.5 \mathrm{mmol})$ in anhydrous benzyl alcohol ( $5 \mathrm{~mL})$ was added to reaction mixture slowly. After stirred for $3 \mathrm{~h}$ at $70^{\circ} \mathrm{C}$, a reaction mixture was filtered under suction, and then the filtrate was washed with diethyl ether $\left(\mathrm{Et}_{2} \mathrm{O}\right)$. The $\mathrm{Et}_{2} \mathrm{O}$ solution evaporated to dryness. The residue was dissolved in $\mathrm{CHCl}_{3}(100 \mathrm{~mL})$ and washed with $10 \%$ aqueous potassium iodide $(20 \mathrm{~mL}$ x 5$)$ and $10 \%$ sodium thiosulfate $(20 \mathrm{~mL})$ then the organic layer was dried $\left(\mathrm{MgSO}_{4}\right)$. After the filtrate was evaporated in vacuo, the residue was subjected to silica gel column chromatography employing hexane-AcOEt to give 4,5-di- $O$-acetyl-2,3-di- $O$-benzyl-D-ribose dibenzyl acetal (530 mg, 87\%). ${ }^{1} \mathrm{H}-\mathrm{NMR}\left(\mathrm{CDCl}_{3}\right): \delta 7.2-7.4(20 \mathrm{H}, \mathrm{m}, \mathrm{Ph}-\mathrm{H}), 5.41\left(1 \mathrm{H}, \mathrm{ddd}, \mathrm{J}_{3,4}=5.5 \mathrm{~Hz}\right.$ $\left.\mathrm{J}_{4,5}=6.3 \mathrm{~Hz}, \mathrm{~J}_{4,5^{\circ}}=2.5 \mathrm{~Hz}, \mathrm{H} 4\right), 4.85\left(1 \mathrm{H}, \mathrm{d}, \mathrm{J}_{1,2}=6.0 \mathrm{~Hz}, \mathrm{H} 1\right), 4.82(1 \mathrm{H}, \mathrm{d}, \mathrm{J}=11.5 \mathrm{~Hz}$, Bn-CH), $4.77(1 \mathrm{H}, \mathrm{d}, \mathrm{J}=11.5 \mathrm{~Hz}, \mathrm{Bn}-\mathrm{CH}), 4.69(1 \mathrm{H}, \mathrm{d}, \mathrm{J}=11.7 \mathrm{~Hz}, \mathrm{Bn}-\mathrm{CH}), 4.66$ (2H, s, Bn-CH x 2), 4.5-4.6 (3H, Bn-CH x 3), $4.48\left(1 \mathrm{H}, \mathrm{dd}, \mathrm{J}_{4,5^{\prime}}=2.5 \mathrm{~Hz}, \mathrm{~J}_{5,5^{\circ}}=12.2 \mathrm{~Hz}, \mathrm{H} 5^{\prime}\right)$, $4.23\left(1 \mathrm{H}, \mathrm{dd}, \mathrm{J}_{4,5}=6.3 \mathrm{~Hz}, \mathrm{~J}_{5,5^{\circ}}=12.2 \mathrm{~Hz}, \mathrm{H} 5\right), 3.94\left(1 \mathrm{H}, \mathrm{dd}, \mathrm{J}_{2,3}=3.4 \mathrm{~Hz}, \mathrm{~J}_{3,4}=5.5 \mathrm{~Hz}\right.$ H3), $3.85\left(1 \mathrm{H}, \mathrm{dd}, \mathrm{J}_{1,2}=6.0 \mathrm{~Hz}, \mathrm{~J}_{2,3}=3.4 \mathrm{~Hz}, \mathrm{H} 2\right), 1.96\left(3 \mathrm{H}, \mathrm{s}, \mathrm{COCH}_{3}\right), 1.91(3 \mathrm{H}, \mathrm{s}$, $\left.\mathrm{COCH}_{3}\right){ }^{13} \mathrm{C}-\mathrm{NMR}\left(\mathrm{CDCl}_{3}\right): \delta 170.62,169.62,138.12,137.98,137.70,137.54,128.35$ 
$128.31,128.27,128.21,128.07,128.03,127.89,127.83,127.68,127.64,127.52,101.83$, 79.74, 77.89, 74.34, 72.71, 71.36, 70.04, 69.92, 63.15, 20.91, 20.74. Anal. calcd for $\mathrm{C}_{37} \mathrm{H}_{40} \mathrm{O}_{8}: \mathrm{C}, 72.35 ; \mathrm{H}, 6.58$. Found: C, 72.20; H, 6.68.

2,3-Di-O -benzyl-D-ribose dibenzyl acetal (4-Bn)

${ }^{1} \mathrm{H}-\mathrm{NMR}\left(\mathrm{CDCl}_{3}\right): \delta$ 7.2-7.4 (20H, m, Ph-H), $4.90\left(1 \mathrm{H}, \mathrm{d}, \mathrm{J}_{1,2}=5.7 \mathrm{~Hz}, \mathrm{H} 1\right), 4.81(1 \mathrm{H}, \mathrm{d}$, $\mathrm{J}=11.5 \mathrm{~Hz}, \mathrm{Bn}-\mathrm{CH}), 4.76(1 \mathrm{H}, \mathrm{d}, \mathrm{J}=11.5 \mathrm{~Hz}, \mathrm{Bn}-\mathrm{CH}), 4.72(1 \mathrm{H}, \mathrm{d}, \mathrm{J}=11.6 \mathrm{~Hz}, \mathrm{Bn}-\mathrm{CH})$, $4.70(1 \mathrm{H}, \mathrm{d}, \mathrm{J}=11.7 \mathrm{~Hz}, \mathrm{Bn}-\mathrm{CH}), 4.65(1 \mathrm{H}, \mathrm{d}, \mathrm{J}=11.7 \mathrm{~Hz}, \mathrm{Bn}-\mathrm{CH}), 4.58(1 \mathrm{H}, \mathrm{d}, \mathrm{J}=11.5$ Hz, Bn-CH), $4.57(1 \mathrm{H}, \mathrm{d}, \mathrm{J}=11.6 \mathrm{~Hz}, \mathrm{Bn}-\mathrm{CH}), 4.48(1 \mathrm{H}, \mathrm{d}, \mathrm{J}=11.5 \mathrm{~Hz}, \mathrm{Bn}-\mathrm{CH}), 3.94$ $\left(1 \mathrm{H}, \mathrm{dd}, \mathrm{J}_{1,2}=5.7 \mathrm{~Hz}, \mathrm{~J}_{2,3}=2.7 \mathrm{~Hz}, \mathrm{H} 2\right), 3.90(1 \mathrm{H}, \mathrm{m}, \mathrm{H} 4), 3.82\left(1 \mathrm{H}, \mathrm{dd}, \mathrm{J}_{2,3}=2.7 \mathrm{~Hz}, \mathrm{~J}_{3,4}\right.$ $=7.4 \mathrm{~Hz}, \mathrm{H} 3), 3.73\left(1 \mathrm{H}, \mathrm{ddd}, \mathrm{J}_{4,5^{\circ}}=2.7 \mathrm{~Hz}, \mathrm{~J}_{5,5^{\prime}}=11.25 \mathrm{~Hz}, \mathrm{~J}_{5^{\prime}, 5 \mathrm{OH}}=6.5 \mathrm{~Hz}, \mathrm{H} 5^{\prime}\right), 3.65$ $\left(1 \mathrm{H}, \mathrm{ddd}, \mathrm{J}_{4,5}=4.7 \mathrm{~Hz}, \mathrm{~J}_{5,5^{\circ}}=11.3 \mathrm{~Hz}, \mathrm{~J}_{5,5 \mathrm{OH}}=6.5 \mathrm{~Hz}, \mathrm{H} 5\right), 3.09(1 \mathrm{H}, \mathrm{bd}, \mathrm{J}=5.7 \mathrm{~Hz}, 4-$ $\mathrm{OH}), 2.08(1 \mathrm{H}, \mathrm{t}, \mathrm{J}=6.5 \mathrm{~Hz}, 5-\mathrm{OH}) .{ }^{13} \mathrm{C}-\mathrm{NMR}\left(\mathrm{CDCl}_{3}\right): \delta 137.93,137.74,137.14,128.43$, $128.38,128.31,127.99,127.90,127.82,127.71,101.70,80.59,79.28,74.25,72.85,71.18$ 70.42, 70.10, 63.86. Anal. calcd for $\mathrm{C}_{33} \mathrm{H}_{36} \mathrm{O}_{6}-1 / 2 \mathrm{H}_{2} \mathrm{O}: \mathrm{C}, 73.72 ; \mathrm{H}, 6.94$. Found: $\mathrm{C}, 73.84$; H, 6.80._

\section{2,3-Di-O -benzyl-D-ribose dimethyl acetal (4-Me)}

Compound 3 (2.60_, $5.32 \mathrm{mmol}$ ) was treated similarly to 4-Bn with methanol to give 4-Me $(1.76 \mathrm{~g}, 96 \%)$ as a foam. ${ }^{1} \mathrm{H}-\mathrm{NMR}\left(\mathrm{CDCl}_{3}\right): \delta 7.35-7.26(10 \mathrm{H}, \mathrm{m}, \mathrm{Ph}-\mathrm{H}), 4.80(1 \mathrm{H}, \mathrm{d}, \mathrm{J}=$ 11.5 Hz, Bn-H), $4.74(1 \mathrm{H}, \mathrm{d}, \mathrm{J}=11.6 \mathrm{~Hz}, \mathrm{Bn}-\mathrm{H}), 4.66(1 \mathrm{H}, \mathrm{d}, \mathrm{J}=11.4 \mathrm{~Hz}, \mathrm{Bn}-\mathrm{CH}), 4.53$ $(1 \mathrm{H}, \mathrm{d}, \mathrm{J}=11.4 \mathrm{~Hz}, \mathrm{Bn}-\mathrm{CH}), 4.50\left(1 \mathrm{H}, \mathrm{d}, \mathrm{J}_{1,2}=5.8 \mathrm{~Hz}, \mathrm{H} 1\right), 3.89(1 \mathrm{H}, \mathrm{m}, \mathrm{H} 4), 3.82(1 \mathrm{H}, \mathrm{dd}$, $\left.\mathrm{J}_{1,2}=5.8 \mathrm{~Hz}, \mathrm{~J}_{2,3}=2.7 \mathrm{~Hz}, \mathrm{H} 2\right), 3.79-3.74(2 \mathrm{H}, \mathrm{m}, \mathrm{H} 3$ and $\mathrm{H} 5), 3.70-3.65(1 \mathrm{H}, \mathrm{m}, \mathrm{H} 5), 3.47$ $\left(6 \mathrm{H}, \mathrm{s}, \mathrm{OCH}_{3} \times 2\right), 3.22(1 \mathrm{H}, \mathrm{d}, \mathrm{J}=4.6 \mathrm{~Hz}, 4-\mathrm{OH}), 2.21(1 \mathrm{H}, \mathrm{t}, \mathrm{J}=6.5 \mathrm{~Hz}, \mathrm{~J}=6.4 \mathrm{~Hz}, 5-$ 
$\mathrm{OH}) .{ }^{13} \mathrm{C}-\mathrm{NMR}\left(\mathrm{CDCl}_{3}\right): \delta 137.98,128.45,128.40,128.03,127.85,127.82,105.30,79.99$, 79.40, 74.28, 73.06, 71.17, 63.96, 56.32, 55.92. Anal. calcd for $\mathrm{C}_{21} \mathrm{H}_{28} \mathrm{O}_{6}: \mathrm{C}, 67.00 ; \mathrm{H}$, 7.50. Found: C, 66.90; H, 7.52. 